\title{
Classification of P300 component using a Riemannian ensemble approach
}

\author{
Dominik Krzemiński ${ }^{1}$, Sebastian Michelmann ${ }^{2}$, Matthias Treder ${ }^{3}$, and \\ Lorena Santamaria $^{1}$ \\ 1 CUBRIC, School of Psychology, Cardiff University, Cardiff CF24 4HQ, United \\ Kingdom \\ KrzeminskiDK@cardiff .ac.uk \\ 2 Princeton Neuroscience Institute, Princeton University, Princeton NJ 08544,USA \\ 3 School of Computer Science \& Informatics, Cardiff University, Cardiff CF24 3AA, \\ United Kingdom
}

\begin{abstract}
We present a framework for P300 ERP classification on the 2019 IFMBE competition dataset using a combination of a Riemannian geometry and ensemble learning. Covariance matrices and ERP prototypes are extracted after the EEG is passed through a filter bank and an ensemble of LDA classifiers is trained on subsets of channels, trials, and frequencies. The model selects a final class based on maximum probability of evidence from all ensembles. Our pipeline achieves an average classification accuracy of $81.2 \%$ on the test set.
\end{abstract}

Keywords: brain-computer interface, ERP, P300, classification, Riemannian geometry, ensemble learning, LDA, logistic regression

\section{Introduction}

The P300 is a positive deflection in the event-related potential (ERP) focused on centro-parietal electrode sites, usually observed around $300 \mathrm{~ms}$ after a stimuli was presented [10]. It typically follows the occurrence of a rare or unexpected sensory stimulus. Unlike the mismatch negativity, it is mediated by the conscious deployment of attention. For this reason, it has been one of the first control paradigms to be used in brain-computer interfaces [7].

The classical approach to P300 feature extraction is the use of spatio-temporal features $[7,11]$. Recently, the Riemannian framework - originally used for oscillatory (e.g. motor imagery) data - has been applied to ERP classification [8]. The framework was developed as a method to directly use covariance matrices as features for classification. Symmetric positive definite (SPD) matrices such as non-singular covariance matrices form a Riemannian manifold in the space of matrices. Since the space is non-linear, it has been suggested to first map the covariance matrices into the linear tangent space with respect to a reference SPD matrix, usually the geometric mean of the training data. The approach outperformed state-of-the-art methods such as the Common Spatial Patterns [3]. 
In this paper, we combine the Riemannian framework with the ensemble learning approaches. A separate classifier was constructed for each subject and session according to the following pipeline. First, the EEG is bandpass filtered using different frequency bands in the range 1-20 Hz. Second, ERP templates are extracted and concatenated with the covariance matrix of an individual trial. Third, covariance matrices are mapped into tangent space using a Riemannian approach. Fourth, an ensemble of Linear Discriminant Analysis (LDA) classifiers with shrinkage [5] or Logistic Regression classifiers is constructed using random subsets of features and trials.

\section{Methods}

The data was provided as part of the 2019 IFMBE scientific challenge competition organized by the 15th Mediterranean Conference on Medical and Biological Engineering and Computing (Medicon 2019). The dataset represents the 8-channels EEG recordings of a feasibility clinical trial to train youngsters with Autism Spectrum Disorder (ASD) to follow the social cues $[1,2]$. It consists of 7 sessions divided into training and test subsets each. In our analysis we refer to the test data as a set consisting of testing part of sessions 4-7 (as used in the competition evaluation). The task of a participant was to count how many times one out of eight objects was highlighted in a virtual environment [1].

All analyses were performed in MATLAB (Natick, USA). The code to reproduce our results is available on the public code repository ${ }^{1}$.

We denote the $i$-th trial of a multivariate $N$-channels dataset measured at a set of time instants $t$ as $\mathbf{x}_{i}(t)=\left[x_{1}(t), x_{2}(t), \ldots, x_{n}(t)\right]^{T} \in \mathbb{R}^{N}$, where for $t=$ $1,2, \ldots, T$ the data can be considered as a wide-sense static data array. Collecting all time points for the $i$-th trial in a matrix $\mathbf{X}_{i} \in \mathbb{R}^{N \times T}$ and assuming zero-mean data, this process can be extensively expressed by its spatial covariance matrix as $\mathbf{C}_{i}=1 /(T-1) \mathbf{X} \mathbf{X}^{T}$. A scheme of the pipeline to obtain the features for the classifier assemble is presented on Figure 1.

Several pipelines were constructed with different combinations of filter frequencies and classifiers. However, they all followed the same general structure. First, the data was bandpass filtered and segmented into seven different combinations (red dotted rectangle of Figure 1) to find the most discriminating features for the classifier. Two different filters were selected (1-20 Hz or 1-8 Hz) and two variations of trial length (whole signal or the first $600 \mathrm{~ms}$ after stimuli onset). Additionally, three subsets of electrodes were chosen (all, central or posterior electrodes). The combination of these 3 possibilities (number of channels, window length and filter band) resulted in seven inputs. Lastly, the eighth combination added was the raw signal where no filtering or windowing was performed.

Then, ERP prototypes were created by calculating the ERP for each channel [4]. For robustness, a trimmed mean discarding the 5\% largest and 5\% smallest values was used. ERP prototypes were then concatenated to the single-trial

\footnotetext{
${ }^{1}$ https://github.com/dokato/bci-challange
} 

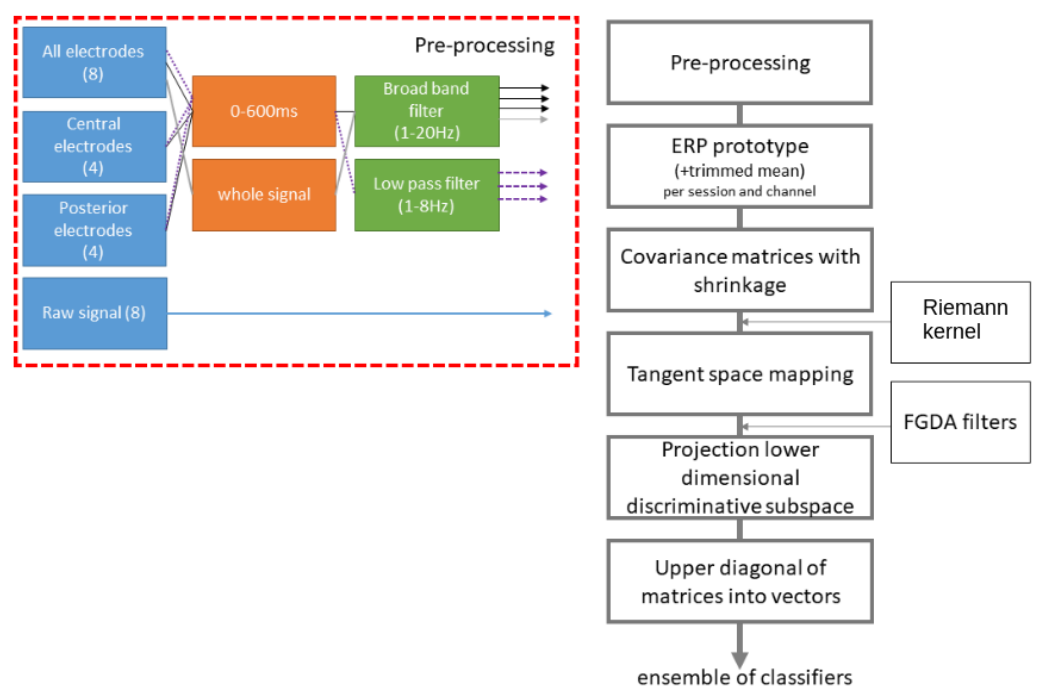

Fig. 1. Depiction of the classification pipeline. The left side (red dotted rectangle) expands on the details of the pre-processing pipeline. The right side (grey rectangles) traces all the steps of the pipeline from pre-processing and feature extraction to classifier training.

data, effectively doubling the number of channels. Regularized covariance matrices were calculated using a shrinkage approach [5]. In shrinkage, the empirical covariance matrix $\mathbf{C}$ is replaced by $\mathbf{C}_{\text {shrink }}=(1-\gamma) \mathbf{C}+\gamma \nu \mathbf{I}$, where $\gamma \in[0,1]$ is a regularization parameter, $\mathbf{I}$ is the identity matrix, and $\nu=\operatorname{trace}(\mathbf{C})$. The Ledoit-Wolf estimate was used to estimate the optimal $\gamma$ [9].

The resultant covariance matrices were projected into the tangent space of a reference matrix. The reference matrix was calculated as the Riemannian geometric mean of the training covariance matrices [3,6]. Next, Fisher Geodesic Discriminant Analysis (FGDA) was used to project the matrices in tangent space to a lower-dimensional discriminative subspace [3]. The resultant projections were flattened to vectors and used as input features to an ensemble learning algorithm as described in the next section.

\section{Results}

We tested several combinations of classifiers and ensembles. The outcomes of classification were tested on a subset of the data from sessions $4-7$. The details of the strategies are listed below.

- Ensemble of data features - 8 different signal features were extracted as follows: (1) All electrodes with broad band frequency spectrum $(1-20 \mathrm{~Hz}) ;(2)$ 
Table 1. Ensemble models' parameters and their accuracies on the test set.

\begin{tabular}{|l|l|c|}
\hline Classifier & Evidence & Accuracy \\
\hline LDA & majority vote & $73.6 \%$ \\
\hline LogReg & majority vote & $69.8 \%$ \\
\hline MDM & majority vote & $71.0 \%$ \\
\hline LDA & probability & $\mathbf{8 1 . 2 \%}$ \\
\hline LogReg & probability & $78.8 \%$ \\
\hline MDM & probability & $80.5 \%$ \\
\hline
\end{tabular}

Central electrodes $(\mathrm{C} 3, \mathrm{Cz}, \mathrm{C} 4, \mathrm{CPz})$ with broad band frequency spectrum; (3) Posterior electrodes (P3, Pz, P4, POz) with broad band frequency spectrum; (4) All electrodes with low-pass filtered signal $(1-8 \mathrm{~Hz})$; (5) Central electrodes with low-pass filtered signal; (6) Posterior electrodes with lowpass filtered signal; (7) All electrodes with broad band frequency spectrum. (8) Raw signal. In the features from (1) to (6) a time window of $0-600 \mathrm{~ms}$ was extracted from the signal. The final result was the combination of all pieces of evidence from 8 classifiers.

- Ensemble of classifiers - ensemble of 400 learners was used taking $40 \%$ of data samples and $60 \%$ of features each. The optimal number of learners was determined using grid search.

- Classifier - type of classifier: linear discriminant analysis (LDA), logistic regression ( $\log R e g)$, or minimum distance to mean (MDM).

- Evidence accumulation - evidence for voting for a final class was either based on majority vote per one trial in block, or based on accumulated probability of voting for a particular class.

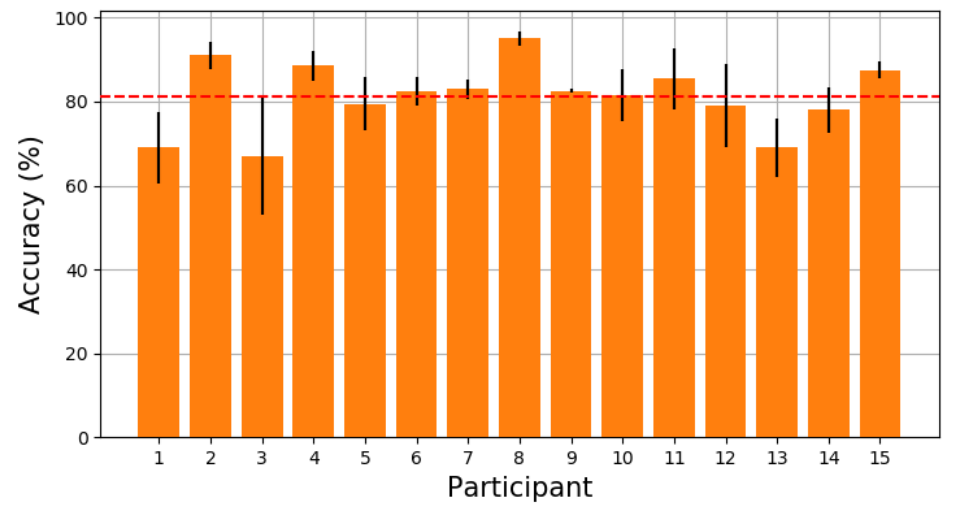

Fig. 2. Performance of the best model per participant of the experiment. Standard error computed over 4 sessions from the test set. The red dashed horizontal line denotes the average accuracy $(81.2 \%)$. 
The performance of a final multi-class accuracy was computed using a ratio of correctly predicted labels to their total number. The best classification accuracy $(81.2 \%)$ was achieved by the model utilizing ensemble of signal features and ensemble of LDA classifiers with cumulative evidence for each of the considered classes. The performance of the model per subject is depicted on Figure 2.

\section{Discussion}

In this study we tested a combination of Riemannian space features extracted from EEG P300 time-series with ensemble approach. The best performance of $81.2 \%$ was achieved by the model combining ensemble of data features with ensemble of LDA classifiers. This result corroborates the feasibility of the proposed approach. Future work will focus on exploring a larger range of classifiers in combination with Riemannian kernels to further increase the accuracy and robustness of the ERP classification.

\section{References}

1. Amaral,C.P., Simões,M.A., Mouga,S., Andrade,J., \& Castelo-Branco, M. (2017). A novel Brain Computer Interface for classification of social joint attention in autism and comparison of 3 experimental setups: A feasibility study. Journal of Neuroscience Methods, 290, 105115.

2. Amaral, C.P., Mouga, S., Simões, M., Pereira, H. C., Bernardino, I., Quental, H. \& Castelo-Branco, M. (2018). A Feasibility Clinical Trial to Improve Social Attention in Autistic Spectrum Disorder (ASD) Using a Brain Computer Interface. Frontiers in Neuroscience, 12(July), 113.

3. Barachant, A., Bonnet, S., Congedo, M., \& Jutten, C. (2010). Riemannian geometry applied to BCI classification. International Conference on Latent Variable Analysis and Signal Separation, 629-636.

4. Barachant, A. \& Congedo, M. (2014). A Plug\&Play P300 BCI Using Information Geometry. arXiv preprint: https://arxiv.org/pdf/1409.0107.pdf .

5. Blankertz, B., Lemm, S., Treder, M., Haufe, S., \& Mller, K. R. (2011). Single-trial analysis and classification of ERP components - A tutorial. NeuroImage, 56(2), 814825 .

6. Congedo, M., Barachant, A., \& Bhatia, R. (2017). Riemannian geometry for EEGbased brain-computer interfaces; a primer and a review. Brain-Computer Interfaces, 120.

7. Farwell, L. \& Donchin, E. (1988). Talking Off the Top of Your Head. Electroencephalography and Clinical Neurophysiology, 70(6), 510523.

8. Korczowski, L., Congedo, M., \& Jutten, C. (2015). Single-trial classification of multiuser P300-based Brain-Computer Interface using Riemannian geometry. In 2015 37th Annual International Conference of the IEEE Engineering in Medicine and Biology Society (EMBC) (pp. 17691772). IEEE.

9. Ledoit, O., \& Wolf, M. (2003). Honey, I shrunk the sample covariance matrix. Economics Working Papers. Retrieved from https://ideas.repec.org/p/upf/upfgen/691. html. 
10. Linden, D. E. J. (2005). The p300: where in the brain is it produced and what does it tell us? The Neuroscientist : A Review Journal Bringing Neurobiology, Neurology and Psychiatry, 11(6), 563576.

11. Treder, M. S., Purwins, H., Miklody, D., Sturm, I., \& Blankertz, B. (2014). Decoding auditory attention to instruments in polyphonic music using single-trial EEG classification. Journal of Neural Engineering, 11(2), 026009. 